Research Article

\title{
A Novel \\ Fractional-Discrete-Cosine-Transform-Based Reversible Watermarking for Healthcare Information Management Systems
}

\author{
Lu-Ting Ko, ${ }^{1}$ Jwu-E Chen, $^{1}$ Yaw-Shih Shieh, ${ }^{2}$ \\ Massimo Scalia, ${ }^{3}$ and Tze-Yun Sung ${ }^{2}$ \\ ${ }^{1}$ Department of Electrical Engineering, National Central University, Chungli 320-01, Taiwan \\ ${ }^{2}$ Department of Electronics Engineering, Chung Hua University, Hsinchu 300-12, Taiwan \\ ${ }^{3}$ Department of Mathematics "Guido Castelnuovo", Sapienza University of Rome, Piazzale Aldo Moro 2, \\ 00185 Rome, Italy
}

Correspondence should be addressed to Tze-Yun Sung, bobsung@chu.edu.tw

Received 7 November 2011; Accepted 30 November 2011

Academic Editor: Ming Li

Copyright (C) 2012 Lu-Ting Ko et al. This is an open access article distributed under the Creative Commons Attribution License, which permits unrestricted use, distribution, and reproduction in any medium, provided the original work is properly cited.

\begin{abstract}
Digital watermarking is a good tool for healthcare information management systems. The wellknown quantization-index-modulation- (QIM-) based watermarking has its limitations as the host image will be destroyed; however, the recovery of medical image is essential to avoid misdiagnosis. A transparent yet reversible watermarking algorithm is required for medical image applications. In this paper, we propose a fractional-discrete-cosine-transform- (FDCT-) based watermarking to exactly reconstruct the host image. Experimental results show that the FDCT-based watermarking is preferable to the QIM-based watermarking for the medical image applications.
\end{abstract}

\section{Introduction}

In the healthcare information systems nowadays, one of the major challenges is a lack of complete access to patients' health information. Ideally, a comprehensive healthcare information system will provide the medical records including health insurance carriers, which are important for clinical decision making. There is sure to be a risk of misdiagnosis, delay of diagnosis, and improper treatments in case of insufficient medical information available [1].

Digital watermarking, which is a technique to embed imperceptible, important data called watermark into the host image, has been applied to the healthcare information 
management systems [2-6]. However, it might cause the distortion problem regarding the recovery of the original host image. In order to protect the host image from being distorted, digital watermarking with legal and ethical functionalities is desirable especially for the medical images applications [7-10]. Specifically, any confidential data such as patients' diagnosis reports can be used as watermark and then embedded in the host image by using digital watermarking with an authorized utilization. Thus, digital watermarking can be used to facilitate healthcare information management systems.

Discrete cosine transform (DCT) has been adopted in various international standards, for example, JPEG, MPEG, and H.264 [11]. The miscellaneous DCT algorithms and architectures have been proposed [12-15]. The fractional discrete cosine transform (FDCT) $[16,17]$, which is a generalized DCT, is yet more applicable in the digital signal processing applications. In this paper, we propose a novel algorithm called the fractional-discretecosine-transform (FDCT-) based watermarking for the healthcare information management applications. In addition, the advantage of FDCT is to take account of the phenomena of image processing $[18,19]$, which is fundamental in nonlinear time series [20,21] and fractal time series [22-25]. The remainder of the paper proceeds as follows. In Section 2, the type I fractional discrete cosine transform is reviewed. Section 3 describes the half discrete cosine transform. The proposed FDCT-based watermarking and experimental results on various medical images are presented in Section 4. The architecture of the half-DCT-based watermarking processor implemented by using FPGA (field programmable gate array) is given in Section 5. The conclusion can be found in Section 4.

\section{Review of Type I Fractional Discrete Cosine Transform}

For the sake of simplicity, let us take the 8-point, type I forward DCT as an example. The corresponding matrix can be expressed as follows $[16,17]$

$$
\mathrm{C}=\left\|\sqrt{\frac{2}{8-1}}\left[k_{m} k_{n} \cos \left(\frac{m n \pi}{8-1}\right)\right]\right\|,
$$

where

$$
\begin{gathered}
k_{m}= \begin{cases}\frac{1}{\sqrt{2}}, & m=1, m=8, \\
1, & 1<m<8,\end{cases} \\
k_{n}= \begin{cases}\frac{1}{\sqrt{2}}, & n=1, n=8, \\
1, & 1<n<8,\end{cases} \\
m=1,2,3, \ldots, 8, \quad n=1,2,3, \ldots, 8 .
\end{gathered}
$$

It can be diagonalized by

$$
\mathbf{C}=\mathbf{U} \Lambda \mathbf{U}^{T},
$$


where $\mathbf{U}$ is an orthonormal matrix obtained from the eigenvectors of $\mathbf{C}$, which is given by

$$
\mathbf{U}=\left[\begin{array}{cccccccc}
-0.0854 & -0.3941 & -0.2698 & 0.3618 & -0.4605 & -0.2208 & 0.5689 & -0.2216 \\
-0.0279 & -0.0878 & 0.2942 & -0.4052 & -0.4381 & 0.3122 & 0.3273 & 0.5868 \\
0.0859 & 0.6984 & -0.2412 & 0.0788 & -0.5416 & -0.3122 & -0.1721 & 0.1418 \\
0.3807 & 0.4165 & 0.4627 & 0.3663 & 0.0691 & 0.3122 & 0.4141 & -0.2442 \\
-0.0454 & 0.3022 & -0.2725 & -0.4071 & 0.4507 & -0.3122 & 0.6014 & 0.0658 \\
-0.4867 & 0.1983 & 0.0220 & -0.4048 & -0.2402 & 0.3122 & 0.0113 & -0.6537 \\
0.6633 & -0.2123 & 0.1550 & -0.4814 & -0.1927 & -0.3122 & -0.0790 & -0.3440 \\
-0.4010 & 0.0090 & 0.6852 & 0.0568 & 0 & -0.6053 & 0 & 0
\end{array}\right]
$$

$\Lambda$ is a diagonal matrix composed of the corresponding eigenvalues, which is given by

$$
\Lambda=\left[\begin{array}{cccccccc}
-1 & 0 & 0 & 0 & 0 & 0 & 0 & 0 \\
0 & -1 & 0 & 0 & 0 & 0 & 0 & 0 \\
0 & 0 & -1 & 0 & 0 & 0 & 0 & 0 \\
0 & 0 & 0 & -1 & 0 & 0 & 0 & 0 \\
0 & 0 & 0 & 0 & 1 & 0 & 0 & 0 \\
0 & 0 & 0 & 0 & 0 & 1 & 0 & 0 \\
0 & 0 & 0 & 0 & 0 & 0 & 1 & 0 \\
0 & 0 & 0 & 0 & 0 & 0 & 0 & 1
\end{array}\right]
$$

and $\mathbf{U}^{T}$ is the transpose matrix of $\mathbf{U}$. Based on (2.3), the square of the DCT matrix can be written as

$$
\mathbf{C}^{2}=\mathbf{C} \cdot \mathbf{C}=\mathbf{U} \boldsymbol{\Lambda} \mathbf{U}^{T} \mathbf{U} \boldsymbol{\Lambda} \mathbf{U}^{T}=\mathbf{U} \Lambda^{2} \mathbf{U}^{T}
$$

Similarly, we have

$$
\mathbf{C}^{\alpha}=\mathbf{U} \Lambda^{\alpha} \mathbf{U}^{T}
$$


where

$$
\Lambda^{\alpha}=\left[\begin{array}{cccccccc}
\lambda_{1}^{a} & 0 & 0 & 0 & 0 & 0 & 0 & 0 \\
0 & \lambda_{2}^{a} & 0 & 0 & 0 & 0 & 0 & 0 \\
0 & 0 & \lambda_{3}^{a} & 0 & 0 & 0 & 0 & 0 \\
0 & 0 & 0 & \lambda_{4}^{a} & 0 & 0 & 0 & 0 \\
0 & 0 & 0 & 0 & \lambda_{5}^{a} & 0 & 0 & 0 \\
0 & 0 & 0 & 0 & 0 & \lambda_{6}^{a} & 0 & 0 \\
0 & 0 & 0 & 0 & 0 & 0 & \lambda_{7}^{a} & 0 \\
0 & 0 & 0 & 0 & 0 & 0 & 0 & \lambda_{8}^{a}
\end{array}\right],
$$

$\alpha$ is a real fraction, $\lambda_{n}^{a}=e^{j\left(\theta_{n}+2 \pi q_{n}\right) a}, n=1,2,3, \ldots, 8, \theta_{1}, \theta_{2}, \theta_{3}, \theta_{4}=\pi$ and $\theta_{5}, \theta_{6}, \theta_{7}, \theta_{8}=0, q_{n}$ is an element of generating sequence (GS) $\mathbf{q}=\left(q_{1}, q_{2}, \ldots, q_{8}\right)$, and $q_{n}$ is an integer for $0 \leq q_{n} \leq 7$.

\section{Half Discrete Cosine Transform}

The half-DCT, that is, the FDCT with $\alpha=1 / 2$ is obtained by

$$
\sqrt{\mathbf{C}}=\mathbf{U} \Lambda^{1 / 2} \mathbf{U}^{T}
$$

where

$$
\Lambda^{1 / 2}=\left[\begin{array}{llllllll}
j & 0 & 0 & 0 & 0 & 0 & 0 & 0 \\
0 & j & 0 & 0 & 0 & 0 & 0 & 0 \\
0 & 0 & j & 0 & 0 & 0 & 0 & 0 \\
0 & 0 & 0 & j & 0 & 0 & 0 & 0 \\
0 & 0 & 0 & 0 & 1 & 0 & 0 & 0 \\
0 & 0 & 0 & 0 & 0 & 1 & 0 & 0 \\
0 & 0 & 0 & 0 & 0 & 0 & 1 & 0 \\
0 & 0 & 0 & 0 & 0 & 0 & 0 & 1
\end{array}\right] .
$$

The matrix $\mathbf{z}$, obtained by combining the 8-point half-DCT of $\mathbf{x}$ and $\mathbf{y}$ is defined as

$$
\mathbf{z}=\mathbf{C}_{1} \mathbf{x}-\mathbf{C}_{1} \mathbf{y}=\mathbf{C}_{1} \mathbf{x}+\mathbf{C}_{2} \mathbf{y}
$$

where

$$
\begin{gathered}
\mathbf{C}_{1}=\mathbf{U} \Lambda^{1 / 2} \mathbf{U}^{T}, \\
\mathbf{C}_{2}=-\mathbf{C}_{1}=-\mathbf{U} \boldsymbol{\Lambda}^{(1 / 2)} \mathbf{U}^{T} .
\end{gathered}
$$


$\mathrm{U}$ is the orthonormal matrix given by

$$
\begin{gathered}
\mathbf{U}=\left[\mathbf{u}_{1}, \mathbf{u}_{2}, \ldots, \mathbf{u}_{n}\right], \\
\mathbf{u}_{m} \mathbf{u}_{n}^{T}= \begin{cases}1, & m=n, \\
0, & m \neq n .\end{cases}
\end{gathered}
$$

Let $\mathbf{U}_{n}$ be defined as

$$
\mathbf{U}_{n}=\mathbf{u}_{n} \mathbf{u}_{n}^{T}
$$

we have

$$
\mathbf{U}_{m} \mathbf{U}_{n}=\left(\mathbf{u}_{m} \mathbf{u}_{m}^{T}\right)\left(\mathbf{u}_{n} \mathbf{u}_{n}^{T}\right)= \begin{cases}\mathbf{U}_{n}=\mathbf{u}_{n} \mathbf{u}_{n}^{T}, & m=n \\ 0, & m \neq n\end{cases}
$$

It is noted that $\mathbf{C}_{1}$ and $\mathbf{C}_{2}$ can be rewritten as

$$
\begin{gathered}
\mathbf{C}_{1}=\mathbf{U} \boldsymbol{\Lambda}^{1 / 2} \mathbf{U}^{T}=\mathbf{C}_{R}+j \mathbf{C}_{I}, \\
\mathbf{C}_{2}=-\mathbf{U} \boldsymbol{\Lambda}^{1 / 2} \mathbf{U}^{T}=\mathbf{C}_{I}+j \mathbf{C}_{R},
\end{gathered}
$$

where

$$
\begin{aligned}
& \mathbf{C}_{R}=\mathbf{U}_{1}+\mathbf{U}_{2}+\mathbf{U}_{3}+\mathbf{U}_{4}, \\
& \mathbf{C}_{I}=\mathbf{U}_{5}+\mathbf{U}_{6}+\mathbf{U}_{7}+\mathbf{U}_{8} .
\end{aligned}
$$

According to (3.7), (3.9) and (3.10) we have

$$
\begin{gathered}
\mathrm{C}_{R} \mathbf{C}_{R}=\mathrm{C}_{R}, \\
\mathrm{C}_{I} \mathbf{C}_{I}=\mathrm{C}_{I}, \\
\mathrm{C}_{R} \mathbf{C}_{I}=0, \\
\mathrm{C}_{I} \mathbf{C}_{R}=0, \\
\mathrm{C}_{R}+\mathrm{C}_{I}=\mathbf{I}, \\
\mathrm{C}_{I}-\mathrm{C}_{R}=-\left(\mathbf{U}_{1}+\mathbf{U}_{2}+\mathbf{U}_{3}+\mathbf{U}_{4}\right)+\left(\mathbf{U}_{5}+\mathbf{U}_{6}+\mathbf{U}_{7}+\mathbf{U}_{8}\right)=\mathbf{C} .
\end{gathered}
$$

From (3.11), we have

$$
\mathbf{z}=\left(\mathbf{C}_{R}+j \mathbf{C}_{I}\right) \mathbf{x}+\left(\mathbf{C}_{I}+j \mathbf{C}_{R}\right) \mathbf{y}
$$




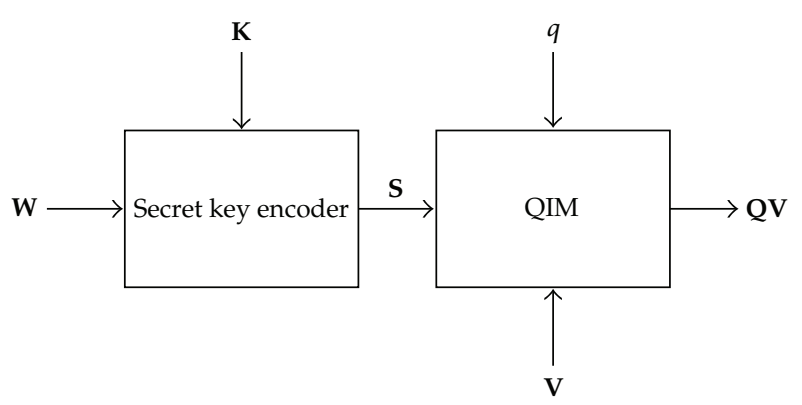

Figure 1: The conventional QIM-based watermarking (W: the watermark, K: the secret key, S: the coded watermark, q: the quantization step, V: the host image, and QV: the watermarked image).

Assume that

$$
\begin{aligned}
\mathbf{y} & =\mathbf{x}+\mathbf{y}^{\prime} \\
\mathbf{z} & =\left(\mathbf{C}_{R}+j \mathbf{C}_{I}\right) \mathbf{x}+\left(\mathbf{C}_{I}+j \mathbf{C}_{R}\right)\left(\mathbf{x}+\mathbf{y}^{\prime}\right) \\
& =\left(\mathbf{C}_{R}+\mathbf{C}_{I}+j \mathbf{C}_{R}+j \mathbf{C}_{I}\right) \mathbf{x}+\left(\mathbf{C}_{I}+j \mathbf{C}_{R}\right) \mathbf{y}^{\prime} \\
& =\mathbf{x}+\mathbf{C}_{I} \mathbf{y}^{\prime}+j\left(\mathbf{x}+\mathbf{C}_{R} \mathbf{y}^{\prime}\right)
\end{aligned}
$$

Thus, $\mathbf{x}$ and $\mathbf{y}$ can be obtained from $\mathbf{z}$ as follows:

$$
\begin{gathered}
\operatorname{Re}\{\mathbf{z}\}-\operatorname{Im}\{\mathbf{z}\}=\left(\mathbf{x}+\mathbf{C}_{I} \mathbf{y}^{\prime}\right)-\left(\mathbf{x}+\mathbf{C}_{R} \mathbf{y}^{\prime}\right)=\left(\mathbf{C}_{I}-\mathbf{C}_{R}\right) \mathbf{y}^{\prime}=\mathbf{C} \cdot \mathbf{y}^{\prime}, \\
\mathbf{y}^{\prime}=\mathbf{C}^{-1} \cdot(\operatorname{Re}\{\mathbf{z}\}-\operatorname{Im}\{\mathbf{z}\}), \\
\mathbf{x}=\operatorname{Re}\{\mathbf{z}\}-\mathbf{C}_{I} \mathbf{y}^{\prime}, \\
\mathbf{x}=\operatorname{Im}\{\mathbf{z}\}-\mathbf{C}_{R} \mathbf{y}^{\prime}, \\
\mathbf{y}=\mathbf{x}+\mathbf{y}^{\prime} .
\end{gathered}
$$

\section{The Proposed Fractional-Discrete-Cosine-Transform-Based Watermarking}

Both transparency and recovery of the host image are required for the medical applications. As the conventional quantization-index-modulation- (QIM-) [26] based watermarking is irreversible, we propose a novel FDCT- based algorithm for reversible watermarking.

\subsection{Quantization Index Modulation}

Figure 1 depicts the conventional QIM-based watermarking [26]. In which, W, K, S, V, and QV denote the watermark, the secret key, the coded watermark, the host image, and the watermarked image, respectively. For the sake of simplicity, let us consider the monochromatic images with 256 grey levels, and the size of the watermark is one-fourth 


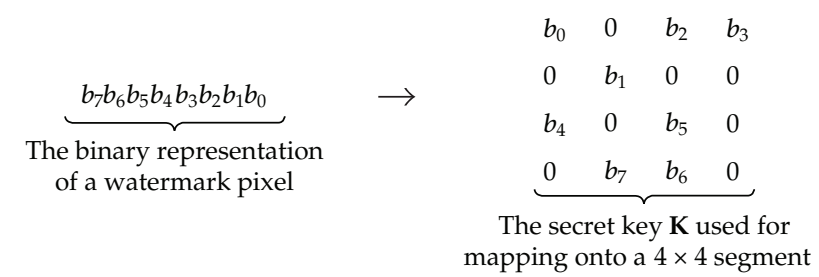

Figure 2: The secret key $\mathbf{K}$ used for mapping the watermark onto the host image.

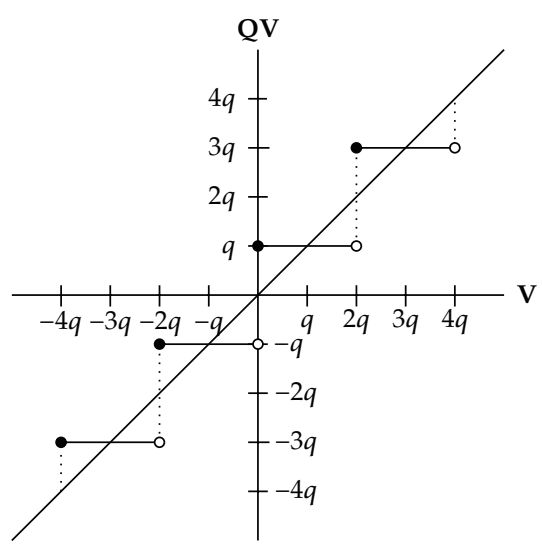

(a)

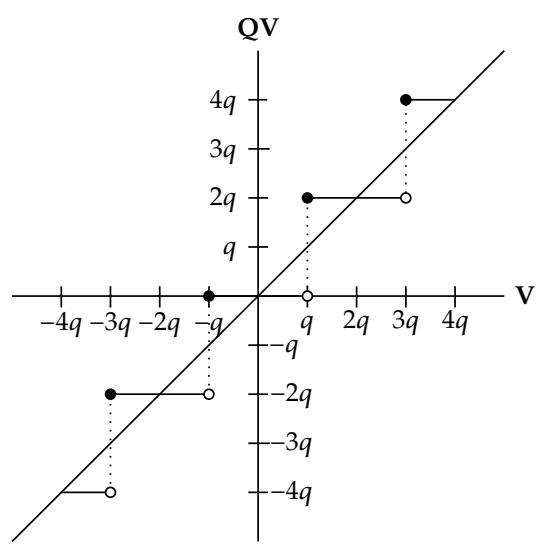

(b)

Figure 3: Operations of the QIM scheme for the coded watermark pixels being (a) bit 1 and (b) bit 0, respectively.

of that of the host image. The secret key is used to map the binary representation of the watermark onto the host image, for example, Figure 2 depicts the binary representation of a watermark pixel that is mapped onto a $4 \times 4$ segment using a given secret key.

Figure 3 shows the operation of the QIM block, in which the grey levels of the host image, $\mathbf{V}$, ranging between $2 c \cdot q$ and $(2 c+1) \cdot q$ will be quantized into $(2 c+1) \cdot q$ if the corresponding pixels of the coded watermark, $\mathbf{S}$, are bit 1 ; otherwise they are quantized into $2 c \cdot q$ if the corresponding pixels are bit 0 . For the grey levels of $\mathbf{V}$ that are between $(2 c+1) \cdot q$ and $(2 c+2) \cdot q$, they will be quantized into $(2 c+1) \cdot q$ or $(2 c+2) \cdot q$ depending on the corresponding pixels of $\mathbf{S}$ being bit 1 or 0 , respectively. Note that $q$ denotes the quantization step, $0 \leq c<255 /(2 \cdot q)$, and $c$ is an integer number.

It is noted that the watermarked image, $\mathbf{Q V}$, can be written as

$$
Q V(i, j)=\left\{\begin{array}{lll}
(2 c+1) q & \text { if } V(i, j) \in((2 c+0.5) q,(2 c+1.5) q], & S(i, j)=1 \\
(2 c) q & \text { if } V(i, j) \in((2 c-0.5) q,(2 c+0.5) q], & S(i, j)=0
\end{array}\right.
$$

where $(i, j)$ denotes the position index of pixels, and the coded watermark, $\mathbf{S}$, can be obtained by

$$
S(i, j)= \begin{cases}1 & \text { if } Q V(i, j) \in((2 d+0.5) q,(2 d+1.5) q] \\ 0 & \text { otherwise }\end{cases}
$$




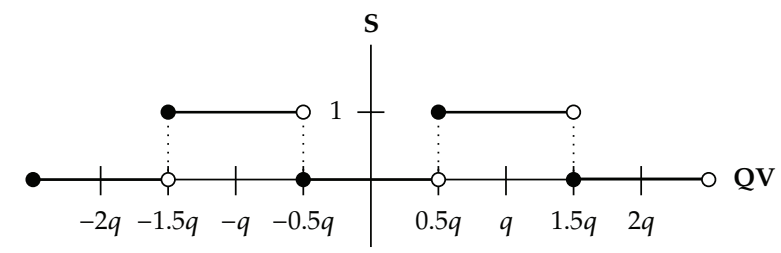

Figure 4: Operations of the inverse QIM scheme for the coded watermark pixels.

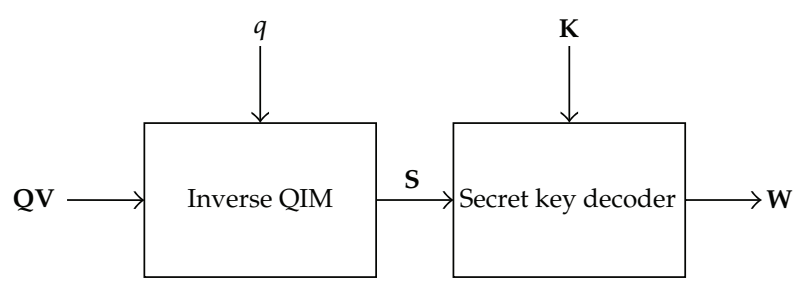

Figure 5: Extraction of the watermark, W, from the watermarked image, $\mathbf{Q V}$, based on the conventional QIM scheme.

as shown in Figure 4. Together with the secret key, K, the watermark, W, can be exactly extracted from the watermarked image, $\mathbf{Q V}$, as shown in Figure 5.

\subsection{Proposed FDCT-Based Watermarking}

According to (3.12), the half-DCT can be used to combine two real valued signals into a single, complex-valued signal. Let $\mathbf{x}$ and $\mathbf{y}$ in (3.12) be the host image and the watermark, respectively, and $\mathbf{z}$ the watermarked image. The watermark and host image can be extracted from $\mathbf{z}$ by using (3.14). Figure 6 depicts the proposed FDCT-based watermarking, where $\mathbf{W}, \mathbf{V}, \mathbf{S}, \mathbf{Q V}, \mathbf{H V}_{R}$, and $\mathbf{H V}_{I}$ are the watermark, the host image, the secret key, the QIM watermarked image, and the watermarked images, $R$ and $I$, respectively. According to (3.12), the half-DCT consists of two matrix multiplications as shown in Figure 7, where $\mathbf{C}_{R}$ and $\mathbf{C}_{I}$ are the half-DCT coefficient matrices for (3.9) and (3.10), respectively.

The original host image, $\mathbf{V}$, and watermark, $\mathbf{W}$, can be exactly reconstructed from the watermarked images: $\mathbf{H} \mathbf{V}_{R}$ and $\mathbf{H} \mathbf{V}_{I}$ as shown in Figures 8 and $\mathbf{9}$, where $\mathbf{C}_{I}$ is the corresponding half-DCT matrix and $\mathrm{C}^{-1}$ is the inverse DCT matrix.

\subsection{Experimental Results on Medical Images}

The proposed FDCT-based watermarking algorithm has been evaluated on various medical images. Figure 10 shows the test $256 \times 256$ images with 256 grey levels, namely, spine, chest, fetus and head obtained by magnetic resonance image (MRI), X-ray, ultrasound, and computed tomography $(\mathrm{CT})$, respectively, which are used as host images. Figure 11 shows the $64 \times 64$ Lena image used as watermark with 256 grey levels.

The peak signal-to-noise ratio (PSNR) is used to evaluate the image quality $[4,8,26]$, which is defined as

$$
\mathrm{PSNR}=20 \log \left(\frac{255}{\sqrt{\mathrm{MSE}}}\right)
$$




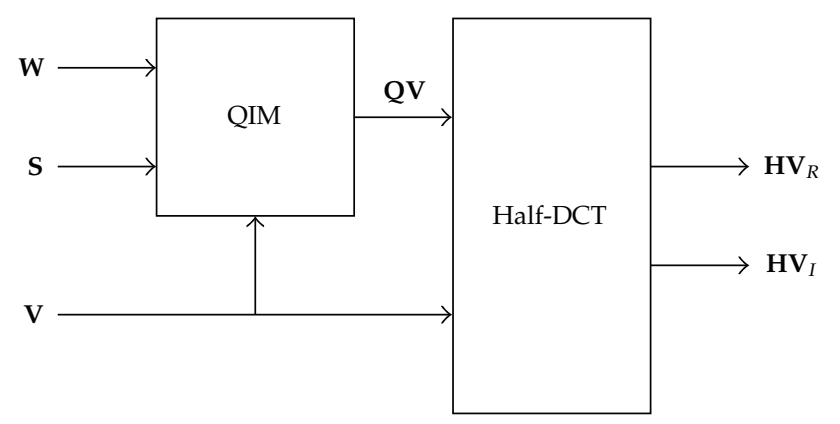

Figure 6: The proposed FDCT-based watermarking (W: the watermark, V: the host image, S: the secret key, $\mathbf{Q V}$ : the QIM watermarked image, and $\mathbf{H V}_{R}$ and $\mathbf{H V}_{I}$ : the watermarked images, $R$ and $I$, resp.).

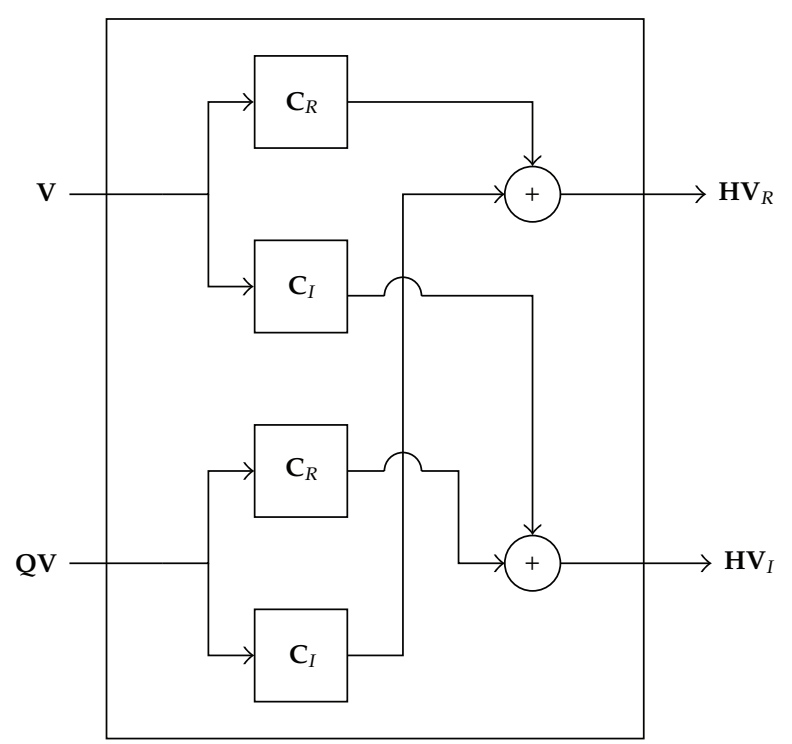

Figure 7: Data flow of the half-DCT operation (V: the host image, QV: the QIM watermarked images, $\mathbf{H V}_{R}$ and $\mathbf{H V}_{I}$ : the watermarked image for real $R$ and imaginary $I$, and $\mathbf{C}_{R}$ and $\mathbf{C}_{I}$ : the corresponding half-DCT matrices.).

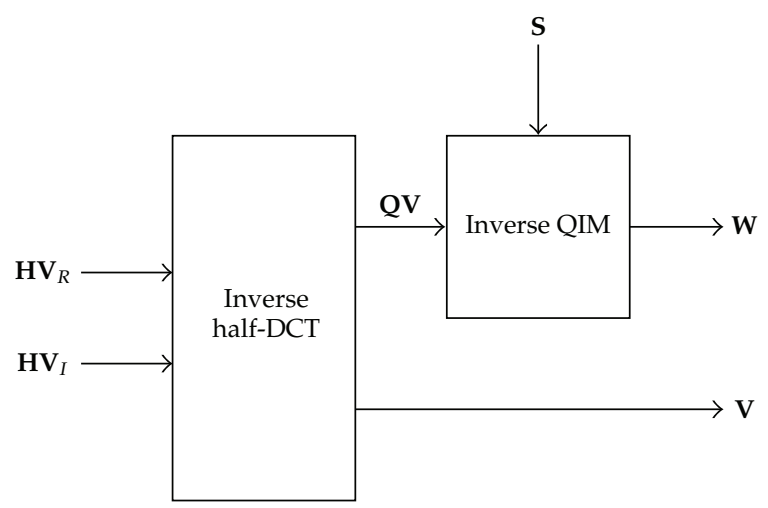

Figure 8: The proposed inverse FDCT-based watermarking for image extraction. 


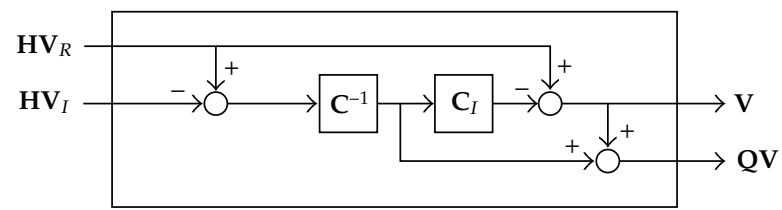

Figure 9: Data flow of the inverse half-DCT operation $\left(\mathbf{C}_{I}\right.$ : the corresponding half-DCT matrix and $\mathbf{C}^{-1}$ : the inverse DCT matrix).

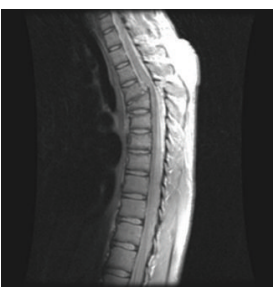

(a)

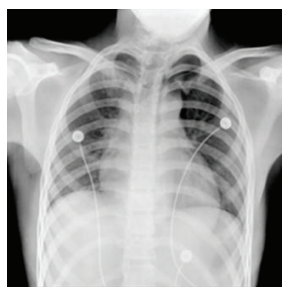

(b)

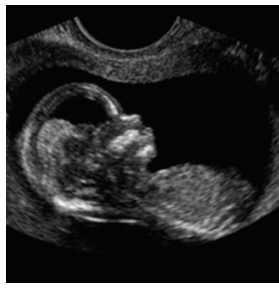

(c)

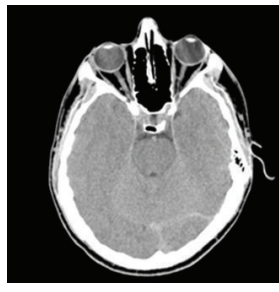

(d)

Figure 10: The $256 \times 256$ host images with 256 grey levels: (a) spine (MRI), (b) chest (X-ray), (c) fetus (ultrasonic), and (d) head (CT).

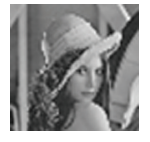

Figure 11: The $64 \times 64$ Lena image with 256 grey levels used as watermarks.

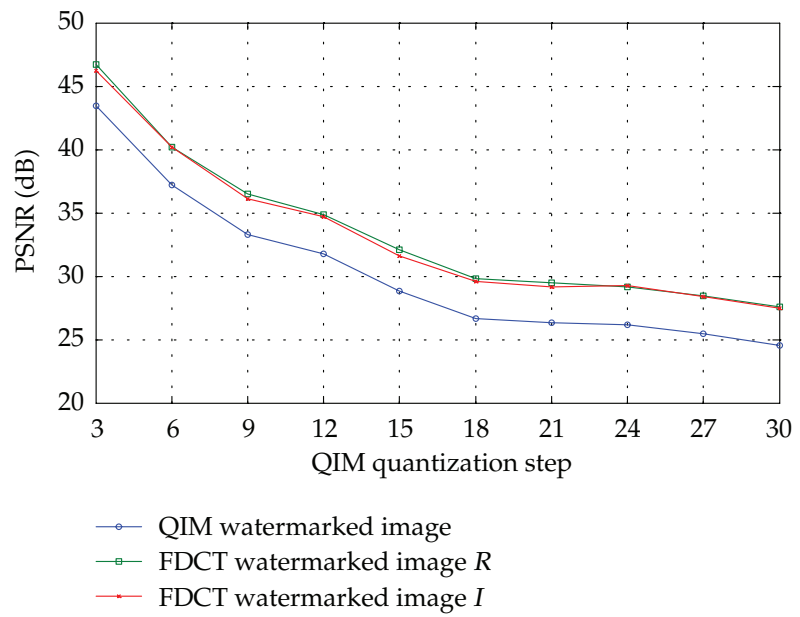

Figure 12: The PSNR of the watermarked image of the spine (MRI) at various QIM quantization steps.

where MSE denotes the mean square error. Figures 12, 13, 14, and 15 show the PSNR of the QIM watermarked image and FDCT watermarked images $R$ and $I$ of spine (MRI), chest (Xray), fetus (ultrasonic), and head (CT) at various QIM quantization steps $q$. Figure 16 shows the QIM watermarked images (first row), the FDCT watermarked images, $R$ (second row) and $I$ (third row), and two extracted watermarks from the $R$ and $I$ watermarked images (fourth row) with QIM quantization step $q=5$. It is noted that the FDCT watermarked images are more transparent than conventional QIM watermarked images, and the block effect of the 


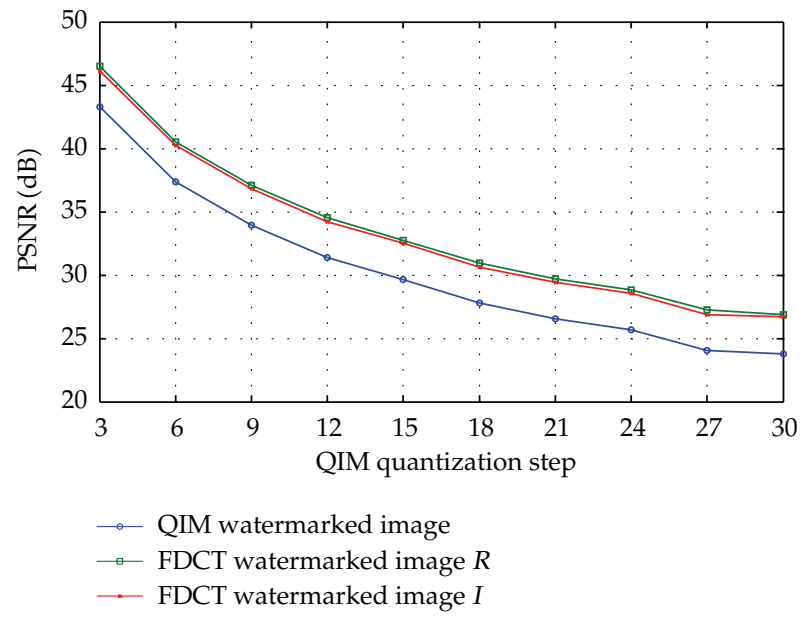

Figure 13: The PSNR of the watermarked image of the chest (X-ray) at various QIM quantization steps.

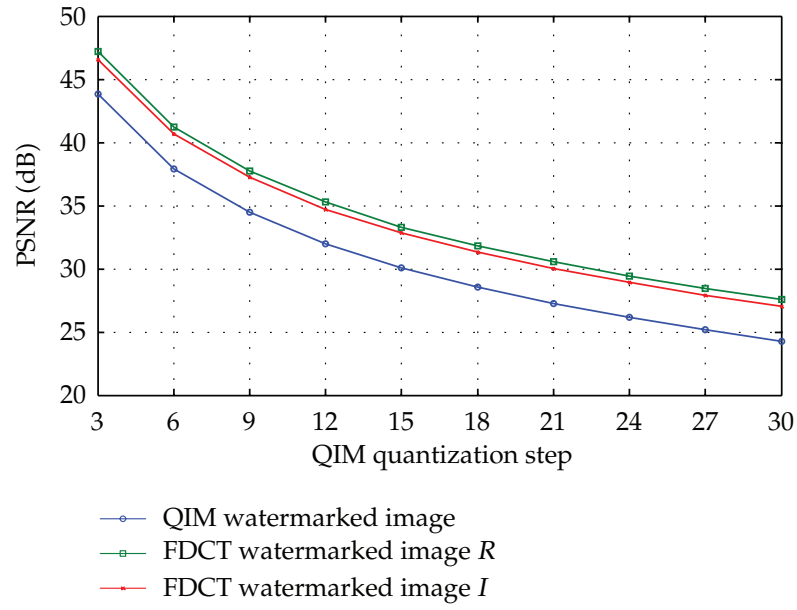

Figure 14: The PSNR of the watermarked image of the fetus (ultrasonic) at various QIM quantization steps.

Table 1: Comparison between this work and the related watermarking algorithms.

\begin{tabular}{lccc}
\hline Items & \multicolumn{3}{c}{ Methods } \\
& Conventional QIM [26] & Nested QIM [8] & FDCT Watermarking \\
\hline Watermarked image transparency & Poor & Good & Better \\
Reversible watermarking & No & Yes & Yes \\
Block effect & Yes & Yes & No \\
\hline
\end{tabular}

FDCT-based watermarking is eliminated. Table 1 shows the comparison between this work and the related watermarking algorithms $[8,26]$. 


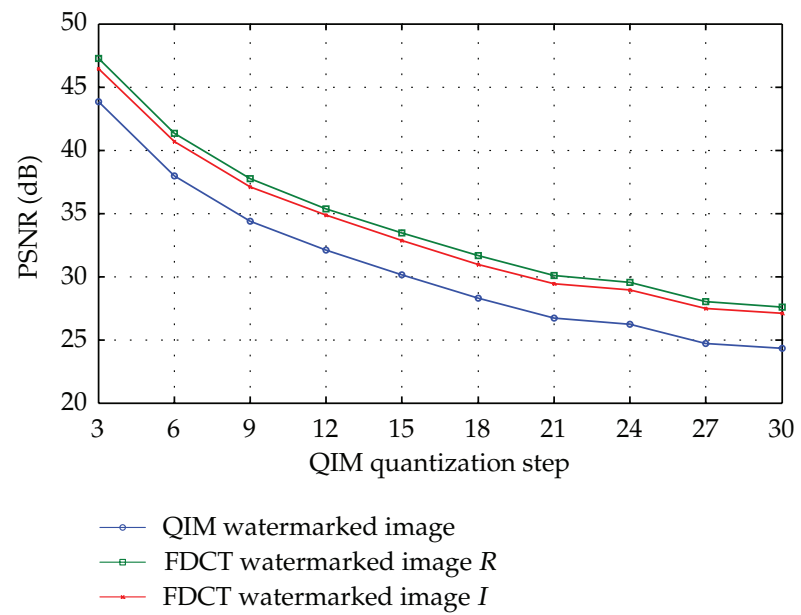

Figure 15: The PSNR of the watermarked image of the head (CT) at various QIM quantization steps.
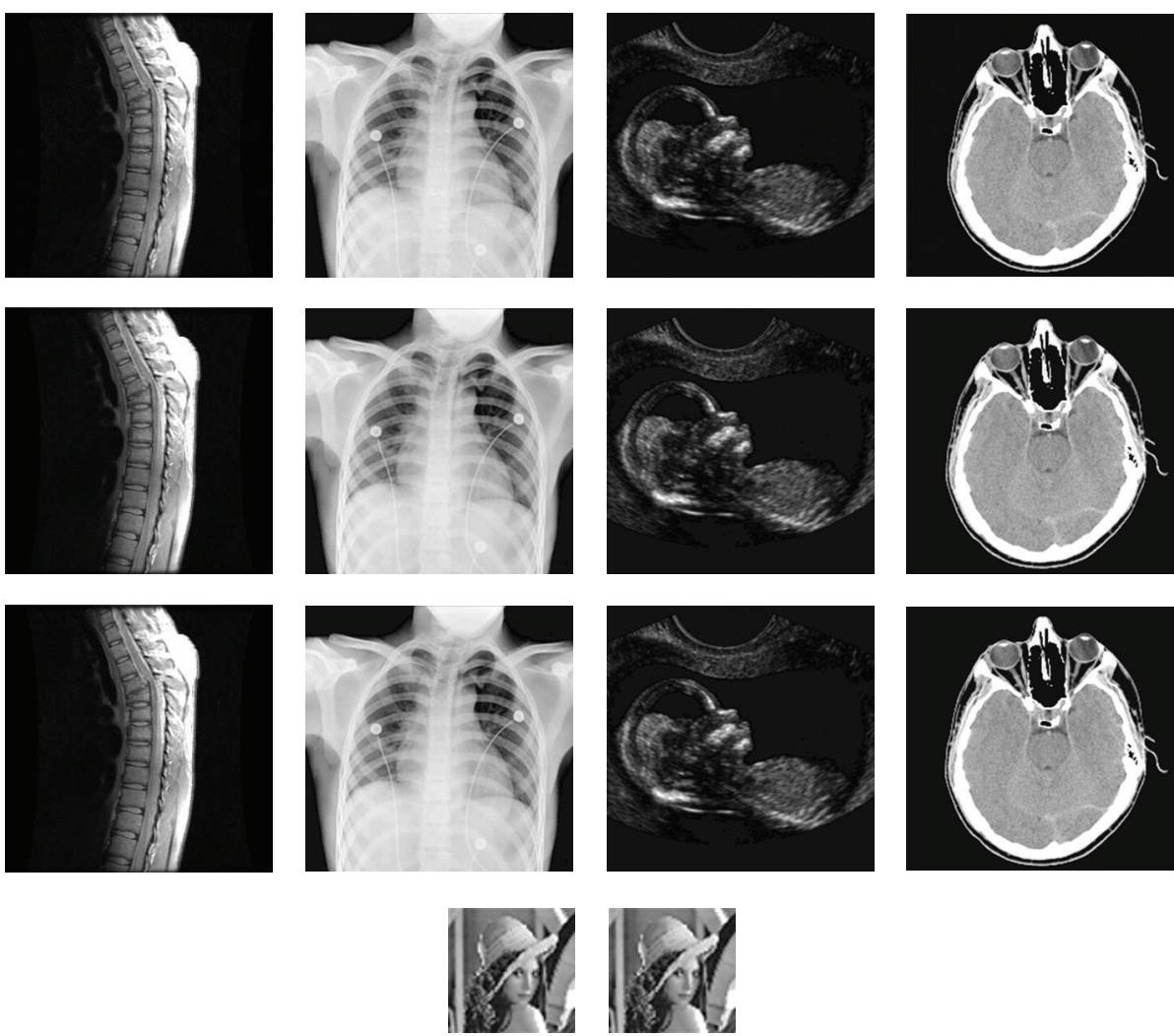

Figure 16: The QIM watermarked images (first row) and the FDCT watermarked images, $R$ (second row) and $I$ (third row), and two extracted watermarks: left one and right one (fourth row) are extracted from the $R$ and $I$ watermarked image, respectively, with QIM quantization step $q=5$. 


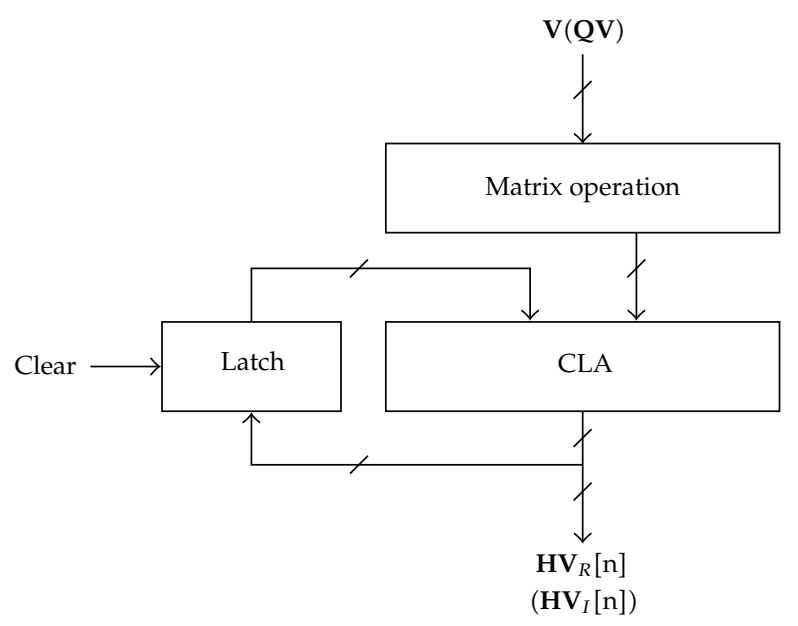

Figure 17: The architecture of the proposed 8-point half-DCT processor.

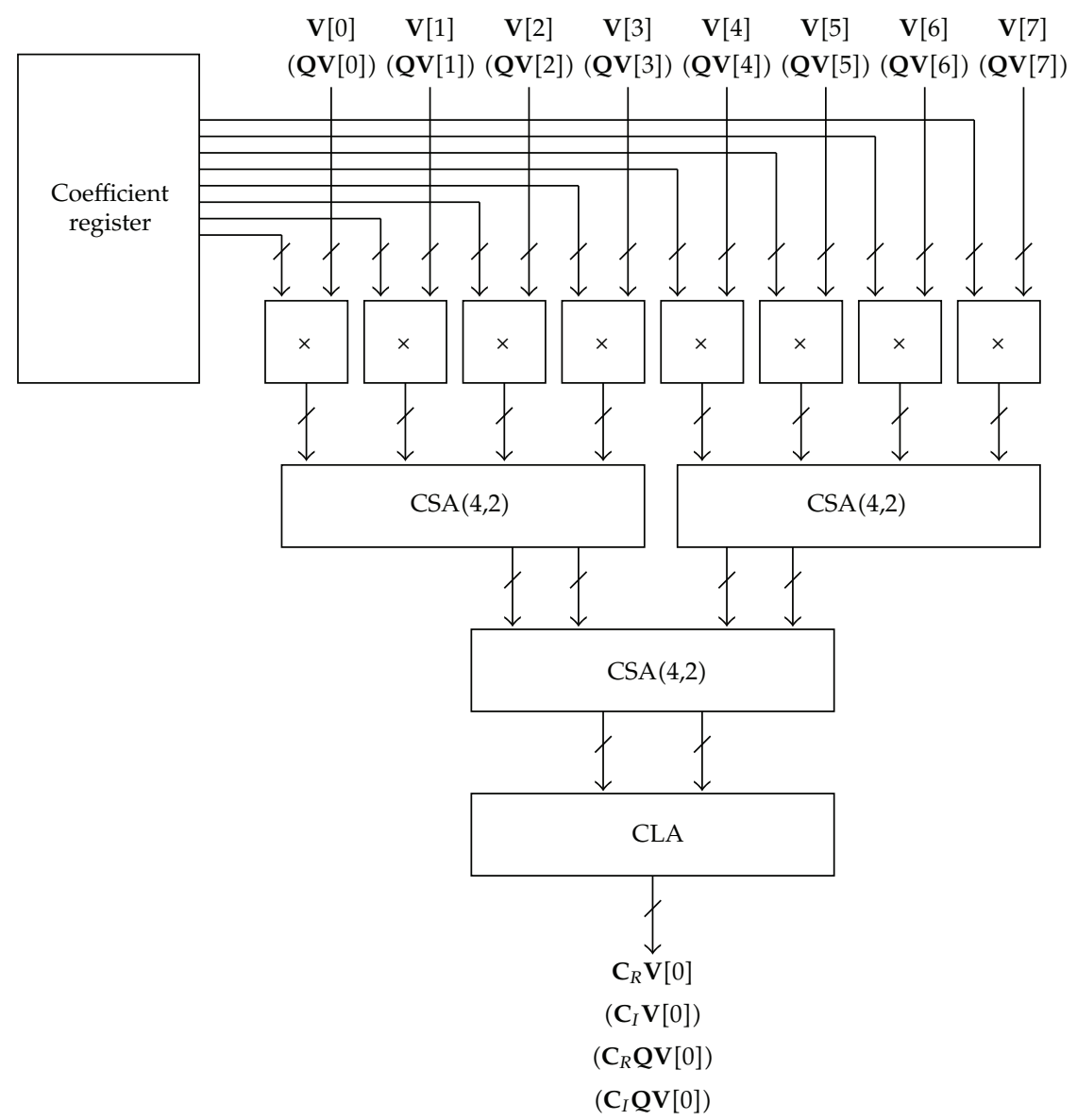

Figure 18: The matrix operation block in the proposed 8-point half-DCT and inverse half-DCT processor. 


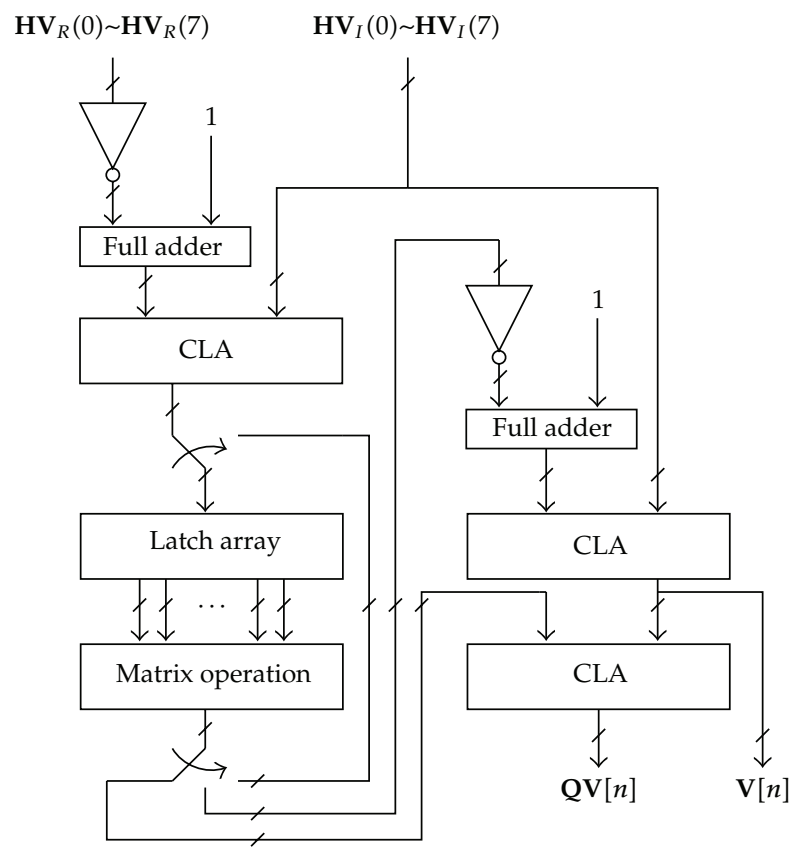

Figure 19: The architecture of the proposed 8-point inverse half-DCT processor.

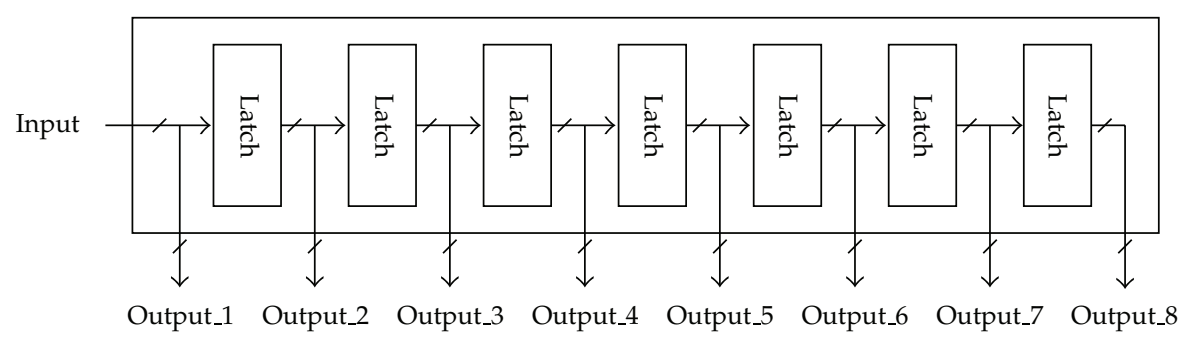

Figure 20: The latch array storing data for matrix operation.

\section{FPGA Implementation of Half-DCT-Based Watermarking Processor}

According to the data flow of the half-DCT shown in Figure 7, the architecture of the proposed 8-point half-DCT processor is shown in Figure 17. In which, the matrix operation block performs the matrix-vector multiplications of $\mathrm{C}_{R} \cdot \mathrm{V}, \mathrm{C}_{I} \cdot \mathrm{V}, \mathrm{C}_{R} \cdot \mathrm{QV}$ and $\mathrm{C}_{I} \cdot \mathrm{QV}$ shown in Figure 18, and the latch and CLA perform the addition operations of $\mathbf{C}_{R} \cdot \mathbf{V}+\mathbf{C}_{I} \cdot \mathbf{Q V}$ and $\mathrm{C}_{I} \cdot \mathrm{V}+\mathrm{C}_{R} \cdot \mathrm{QV}$.

According to the data flow of the inverse half-DCT shown in Figure 9, the architecture of the proposed 8-point inverse half-DCT processor is shown in Figure 19. In which, the matrix operation block performs the matrix-vector multiplications of $\mathbf{C}^{-1} \cdot\left(\mathbf{H V}_{R}-\mathbf{H V}_{I}\right)$ and $\mathbf{C}_{I} \cdot\left(\mathbf{C}^{-1} \cdot\left(\mathbf{H V}_{R}-\mathbf{H V}_{I}\right)\right)$. In the proposed 8-point inverse half-DCT processor as shown in Figure 19, the latch array storing data for matrix operation is shown in Figure 20.

The platform for architecture development and verification has been designed as well as implemented in order to evaluate the development cost. The architecture has been implemented on the Xilinx FPGA emulation board [27]. The Xilinx Spartan-3 FPGA has been 


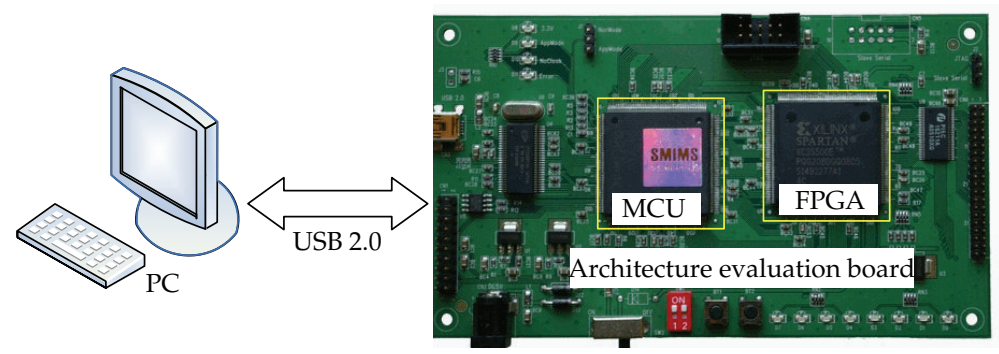

Figure 21: Block diagram and circuit board of the architecture development and verification platform for half-DCT-based watermarking processor.

integrated with the microcontroller (MCU) and I/O interface circuit (USB 2.0) to form the architecture development and verification platform. Figure 21 depicts block diagram and circuit board of the architecture development and evaluation platform, which can perform the prototype of special processor for half-DCT-based watermarking. In the architecture development and evaluation platform, the microcontroller reads data and commands from PC and writes the results back to PC by USB 2.0; the Xilinx Spartan-3 FPGA implements the proposed half-DCT processor. The hardware code written in Verilog is for PC with the ModelSim simulation tool [28] and Xilinx ISE smart compiler [29]. It is noted that the throughput can be improved by using the proposed architecture while the computation accuracy is the same as that obtained by using Matlab technical computing tool [30] with the same word length. Thus, the proposed programmable half-DCT architecture is able to improve the power consumption and computation speed significantly. Moreover, the reusable intellectual property (IP) $8 \times 8$ half-DCT/IDCT core has also been implemented in Verilog hardware description language [31] for the hardware realization. All the control signals are internally generated on-chip. The proposed half-DCT processor provides both high throughput and low gate count.

\section{Conclusion}

In this paper, a novel algorithm called the FDCT-based reversible watermarking has been proposed for medical image watermarking. The transparency of the watermarked image can be increased by taking advantage of the proposed watermarking. As the host image can be exactly reconstructed, it is suitable especially for the medical image applications. In addition, the elimination of block effect avoids detecting QIM coded watermarked image. Thus, the FDCT-based reversible watermarking is preferable to facilitate data management in healthcare information management systems.

\section{References}

[1] H. M. Chao, C. M. Hsu, and S. G. Miaou, "A data-hiding technique with authentication, integration, and confidentiality for electronic patient records," IEEE Transactions on Information Technology in Biomedicine, vol. 6, no. 1, pp. 46-53, 2002.

[2] U. R. Acharya, D. Anand, P. S. Bhat, and U. C. Niranjan, "Compact storage of medical images with patient information," IEEE Transactions on Information Technology in Biomedicine, vol. 5, no. 4, pp. 320 323, 2001.

[3] X. Kong and R. Feng, "Watermarking medical signals for telemedicine," IEEE Transactions on Information Technology in Biomedicine, vol. 5, no. 3, pp. 195-201, 2001. 
[4] A. Giakoumaki, S. Pavlopoulos, and D. Koutsouris, "Multiple image watermarking applied to health information management," IEEE Transactions on Information Technology in Biomedicine, vol. 10, no. 4, pp. 722-732, 2006.

[5] S. Y. Chen and Q. Guan, "Parametric shape representation by a deformable NURBS model for cardiac functional measurements," IEEE Transactions on Biomedical Engineering, vol. 58, no. 3, part 1, pp. 480487, 2011.

[6] M. Li, "Four transformation patterns of two-dimensional function on wavelet basis," Int. J. Engineering and Interdisciplinary Mathematics, vol. 1, no. 2, pp. 107-110, 2009.

[7] L. T. Ko, J. E. Chen, H. C. Hsin, Y. S. Shieh, and T. Y. Sung, "Haar-wavelet-based just noticeable distortion model for transparent watermark," Mathematical Problems in Engineering, vol. 2012, Article ID 635738, 14 pages, 2012.

[8] L. T. Ko, J. E. Chen, Y. S. Shieh, H. C. Hsin, and T. Y. Sung, "Nested quantization index modulation for reversible watermarking and its application to healthcare information management systems," Computational and Mathematical Methods in Medicine, vol. 2012, Article ID 839161, 8 pages, 2012.

[9] S. Y. Chen, H. Tong, Z. Wang, S. Liu, M. Li, and B. Zhang, "Improved generalized belief propagation for vision processing," Mathematical Problems in Engineering, vol. 2011, Article ID 416963, 12 pages, 2011.

[10] S. Y. Chen, H. Tong, and C. Cattani, "Markov models for image labeling," Mathematical Problems in Engineering, vol. 2012, Article ID 814356, 18 pages, 2012.

[11] K. R. Rao and P. Yip, Discrete Cosine Transform: Algorithms, Advantages, Applications, Academic Press, New York, NY, USA, 1990.

[12] L. T. Ko, J. E. Chen, H. C. Hsin, Y. S. Shieh, and T. Y. Sung, "A unified algorithm for subband-based discrete cosine transform," Mathematical Problems in Engineering, vol. 2012, Article ID 912194, 31 pages, 2012.

[13] T. Y. Sung, Y. S. Shieh, and H. C. Hsin, "An efficient VLSI linear array for DCT/IDCT using subband decomposition algorithm," Mathematical Problems in Engineering, vol. 2010, Article ID 185398, 21 pages, 2010.

[14] H. Huang, T. Y. Sung, and Y. S. Shieh, "A novel VLSI linear array for 2-D DCT/IDCT," in Proceedings of the 3rd International Congress on Image and Signal Processing (CISP '10), vol. 8, pp. 3686-3690, Yantai, China, October 2010.

[15] T. Y. Sung, "Memory-efficient and high-performance 2-D DCT and IDCT processors based on CORDIC rotation," WSEAS Transactions on Electronics, vol. 3, no. 12, pp. 565-574, 2006.

[16] S. C. Pei and M. H. Yeh, "The discrete fractional cosine and sine transforms," IEEE Transactions on Signal Processing, vol. 49, no. 6, pp. 1198-1207, 2001.

[17] G. Cariolaro, T. Erseghe, and P. Kraniauskas, "The fractional discrete cosine transform," IEEE Transactions on Signal Processing, vol. 50, no. 4, pp. 902-911, 2002.

[18] Z. Liao, S. Hu, M. Li, and W. Chen, "Noise estimation for single-slice sinogram of low-dose X-Ray computed tomography using homogenous patch," Mathematical Problems in Engineering, vol. 2012, Article ID 696212, 16 pages, 2012.

[19] Z. Liao, S. Hu, D. Sun, and W. Chen, "Enclosed laplacian operator of nonlinear anisotropic diffusion to preserve singularities and delete isolated points in image smoothing," Mathematical Problems in Engineering, vol. 2011, Article ID 749456, 15 pages, 2011.

[20] C. Cattani and A. Kudreyko, "Application of periodized harmonic wavelets towards solution of eigenvalue problems for integral equations," Mathematical Problems in Engineering, vol. 2010, Article ID 570136, 8 pages, 2010.

[21] M. Li and W. Zhao, "Visiting power laws in cyber-physical networking systems," Mathematical Problems in Engineering, vol. 2012, Article ID 302786, 13 pages, 2012.

[22] M. Li, "Fractal time series-a tutorial review," Mathematical Problems in Engineering, vol. 2010, Article ID 157264, 26 pages, 2010.

[23] C. Cattani, "Harmonic wavelet approximation of random, fractal and high frequency signals," Telecommunication Systems, vol. 43, no. 3-4, pp. 207-217, 2010.

[24] M. Li, S. C. Lim, and S. Chen, "Exact solution of impulse response to a class of fractional oscillators and its stability," Mathematical Problems in Engineering, vol. 2011, Article ID 657839, 9 pages, 2011.

[25] E. G. Bakhoum and C. Toma, "Specific mathematical aspects of dynamics generated by coherence functions," Mathematical Problems in Engineering, vol. 2011, Article ID 436198, 10 pages, 2011.

[26] B. Chen and G. W. Wornell, "Quantization index modulation: a class of provably good methods for digital watermarking and information embedding," IEEE Transactions on Information Theory, vol. 47, no. 4, pp. 1423-1443, 2001. 
[27] SMIMS Technology Corp, http://www.smims.com.

[28] Cadence design systems, http://www.cadence.com/products/pages/default.aspx.

[29] Xilinx FPGA products, http://www.xilinx.com/products/.

[30] MATLAB - The language of technical computing, http://www.mathworks.com/products/matlab/ index.html.

[31] D. E. Thomas and P. R. Moorby, The Verilog Hardware Description Language, Baker \& Taylor Books, 5th edition, 2002. 


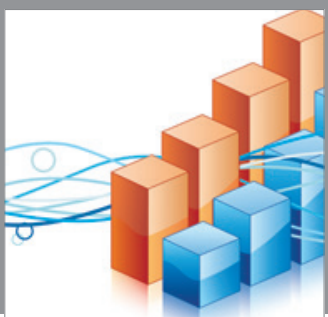

Advances in

Operations Research

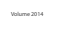

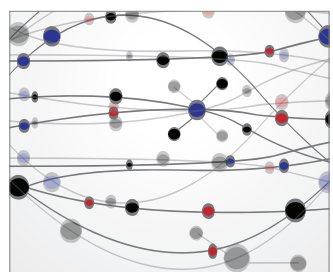

\section{The Scientific} World Journal
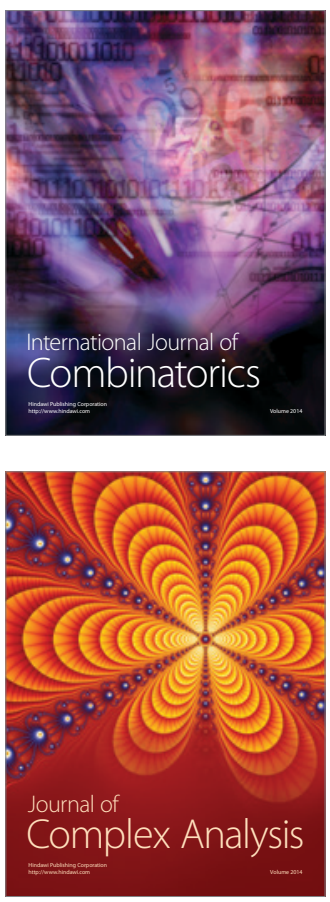

International Journal of

Mathematics and

Mathematical

Sciences
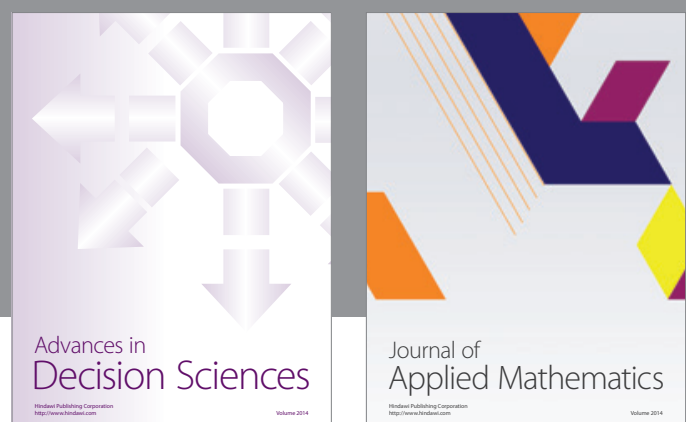

Journal of

Applied Mathematics
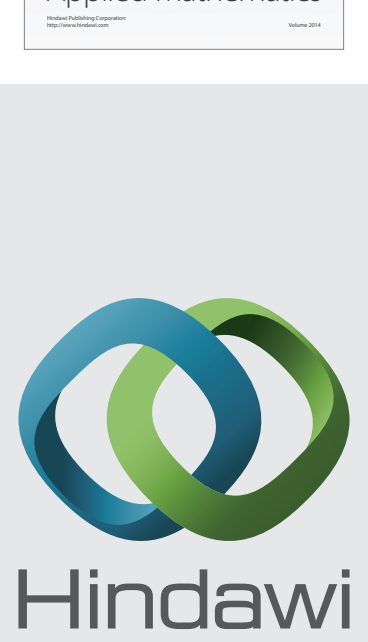

Submit your manuscripts at http://www.hindawi.com
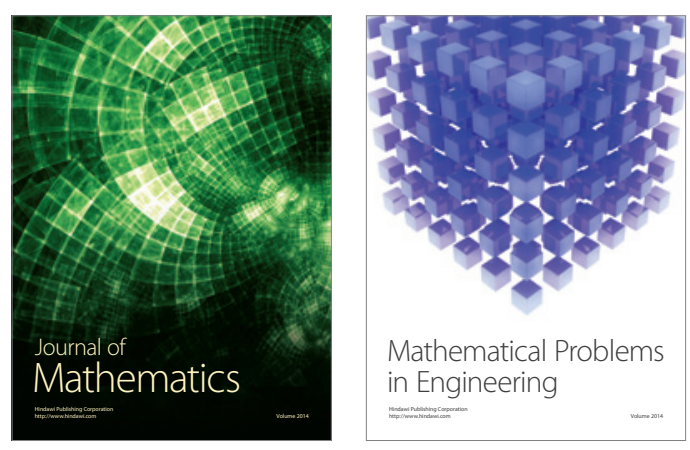

Mathematical Problems in Engineering
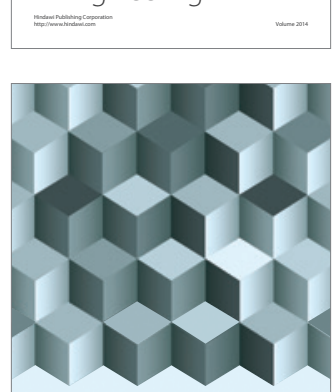

Journal of

Function Spaces
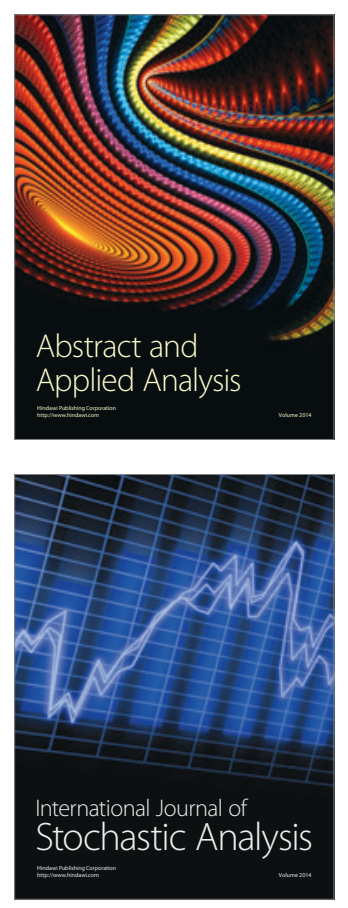

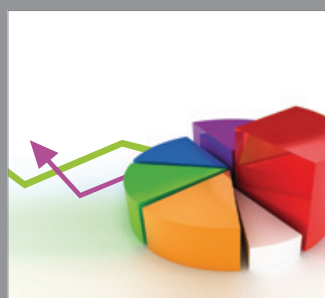

ournal of

Probability and Statistics

Promensencen
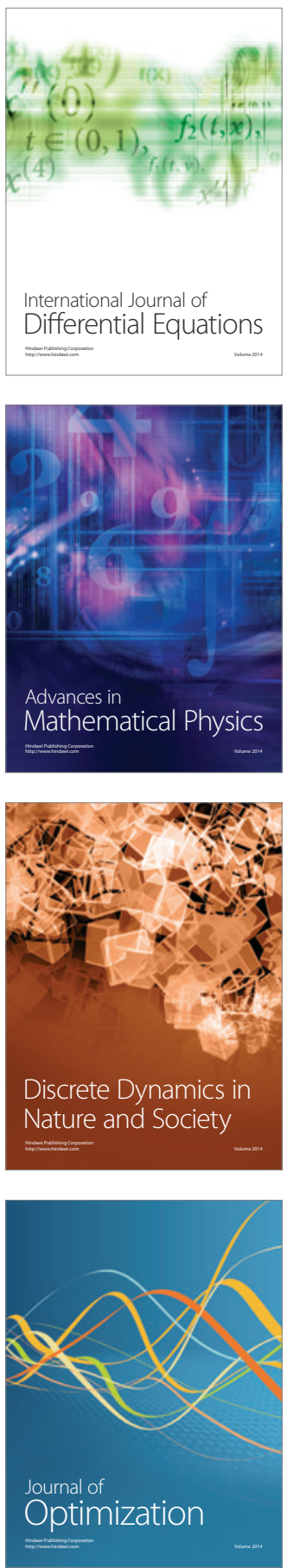